\title{
Correlations Between Slant Wet Delays Measured by Microwave Radiometry
}

\author{
Tobias Nilsson, Lubomir Gradinarsky, Member, IEEE, and Gunnar Elgered
}

\begin{abstract}
We present an analysis of the correlation between the atmospheric slant wet delays in different directions using data from a microwave radiometer. The correlations between wet delays observed in different directions using different temporal constraints are compared to a model derived from theories of turbulence. The agreement between the model and the radiometer data was good, and the average squared difference between zenith mapped slant wet delays could be predicted with an accuracy of $0.01-0.04 \mathrm{~cm}^{2}$. Our analysis shows a large short-term variability which variance has a seasonal dependence of about $\pm 26 \%$, largely depending on the refractivity structure constant $C_{n}$. We also demonstrate how the model can be used to characterize the stability of a microwave radiometer.
\end{abstract}

Index Terms-Atmospheric measurements, global positioning system, microwave radiometry, noise measurement, random media, tomography.

\section{INTRODUCTION}

A TMOSPHERIC variability influences radio wave propagation in the atmosphere. It is often conveniently described by the radio refractive index, and its turbulent structure can be described by models [1]. Turbulence affects many applications using radio waves in earth-space geometries. For example, the integrated effect causes scintillation in satellite communication links [2] and variability in the time of arrival of the navigation signal in a global navigational satellite system (GNSS) [3].

One application for which a good characterization of the atmospheric turbulence may be important is ground-based GPS tomography [4], [5]. The capabilities of using a global positioning system (GPS) to estimate the total amount of integrated zenith water vapor are well accepted and are used in meteorological applications, e.g., see [6]-[8]. As the GPS receivers become more accurate and new GNSS are launched, providing more satellites in the sky, the possibilities to estimate the threedimensional structure of the refractive index and indirectly also the atmospheric water vapor will improve. GPS tomography utilizes a local network of GPS receivers. A discretization of the atmosphere is made by dividing it into finite volume pixels (voxels) in which the refractivity is assumed to be constant. Slant wet delays can be described as a linear combinations of the voxel refractivities. Hence, we can form a linear system

Manuscript received April 30, 2004; revised October 20, 2004. This work was supported by the Remote Sensing Committee of the Swedish National Space Board. The Adlerbertska Research Foundation funded major parts of the radiometer upgrade in 2002-2003.

The authors are with the Onsala Space Observatory, Onsala, Sweden tobias@ oso.chalmers.se; lbg@oso.chalmers.se; kge@oso.chalmers.se).

Digital Object Identifier 10.1109/TGRS.2004.840659

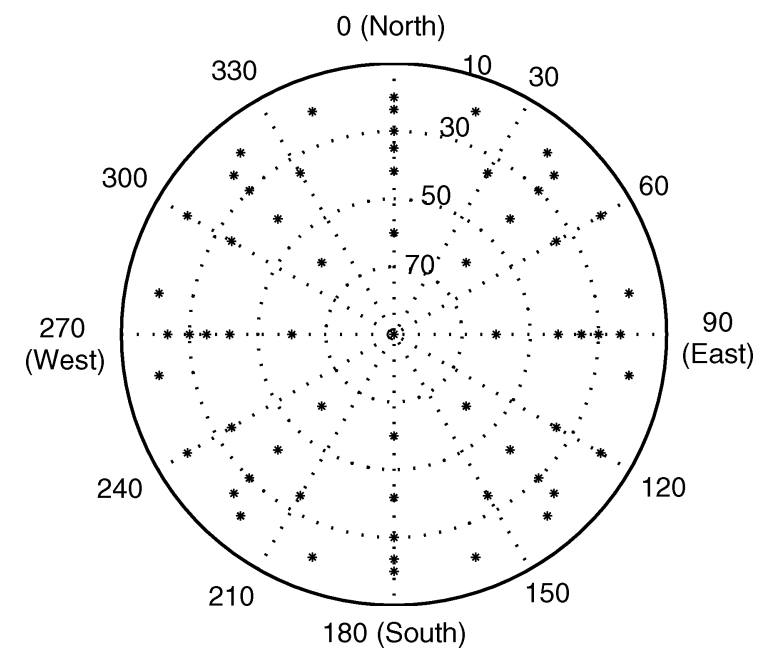

Fig. 1. Polar plot of the direction of the WVR observations used in this work. The lowest elevation angle used in our dataset is $\approx 20^{\circ}$.

of equations where the refractivities of the voxels are the unknowns. However, in general the system is ill conditioned due to the satellite-receiver geometry. This makes it necessary to add additional constraints to make the system solvable. This can be done by using models of the atmospheric turbulence constraining the variability of the refractivity in space and time [5], [9]. One possibility to obtain information in order to develop or validate such models is to use a water vapor radiometer (WVR) to measure slant wet delays during variable weather conditions. WVR data can also be used to validate slant wet delays estimated from GPS data [10].

In this work the WVR at the Onsala Space Observatory was used [11]. It measures the sky brightness temperature at two frequencies, 21.0 and $31.4 \mathrm{GHz}$, with an accuracy of about $1 \mathrm{~K}$. From the measured temperatures it is possible to estimate the wet delay [12]. The half-power beamwidths of the WVR are $6^{\circ}$ for both channels. It has full movability in both azimuth and elevation and has been operating in a "sky-mapping" mode since 1992 , making about 6000-8000 measurements per day. The distribution of the observations in one cycle, which takes about 15 min to complete, is plotted in Fig. 1. During each cycle the WVR makes 64 measurements.

The model used to describe the atmospheric turbulence is presented in Section II. In Section III, we present the results of simulations performed to verify that the WVR data can be used to test the model. The experimental results from the comparison between the WVR data and the model are presented in Section IV, and our conclusions are presented in Section V. 


\section{THEORY}

When traveling through the atmosphere a radio signal will suffer a delay $L_{a t m}$ (measured in unit of length). If the bending of the signal is ignored, this delay can be expressed as [13]

$$
L_{a t m}=\int_{S}(n-1) d s
$$

where $S$ is the path of the signal and $n$ the refractive index of air. This delay can be split into a hydrostatic delay $L_{h}$ and a wet delay $L_{w}$ [12]. If the radio signal is from, for example, a GPS satellite observed at an elevation angle $\epsilon$, the wet delay can be written as $L_{w}(\epsilon)=l m(\epsilon)$, where $l$ is the equivalent zenith wet delay and $m(\epsilon)$ is the (wet) mapping function between zenith and elevation angle $\epsilon$. Letting $\chi$ be the wet refractivity ( $\chi=$ $n_{w}-1$, where $n_{w}$ is the wet component of the refractive index), the equivalent zenith wet delay $l_{i}$ in direction $i$ will be

$$
l_{i}=\frac{1}{m_{i}} \int \chi\left(\mathbf{r}_{i}(z)\right) d s=\int \chi\left(\mathbf{r}_{i}(z)\right) d z
$$

where $\mathbf{r}_{i}(z)$ is the position of the signal at height $z$.

The spatial fluctuations of the wet refractivity in a turbulent atmosphere can be described by a structure function $D_{\chi}[14]$

$$
D_{\chi}\left(\mathbf{r}_{i}, \mathbf{r}_{j}\right)=\left\langle\left(\chi\left(\mathbf{r}_{i}\right)-\chi\left(\mathbf{r}_{j}\right)\right)^{2}\right\rangle=C_{n}^{2} \cdot R^{2 / 3}
$$

where $\langle$.$\rangle denotes expectation value, R=\left|\mathbf{r}_{i}-\mathbf{r}_{j}\right|$, and $C_{n}$ is called the refractivity structure constant.

The correlation between the equivalent zenith wet delays in two different directions ( $i$ and $j$ ) is described by

$$
\left\langle\left(l_{i}-l_{j}\right)^{2}\right\rangle=\left\langle\left(\int\left[\chi\left(\mathbf{r}_{i}(z)\right)-\chi\left(\mathbf{r}_{j}(z)\right)\right] d z\right)^{2}\right\rangle .
$$

In [9] it was shown that this can be rewritten as

$$
\begin{aligned}
\left\langle\left(l_{i}-l_{j}\right)^{2}\right\rangle= & \frac{1}{2} \iint D_{\chi}\left(\mathbf{r}_{i}(z), \mathbf{r}_{j}\left(z^{\prime}\right)\right) d z d z^{\prime} \\
& -\frac{1}{2} \iint D_{\chi}\left(\mathbf{r}_{i}(z), \mathbf{r}_{i}\left(z^{\prime}\right)\right) d z d z^{\prime} \\
& +\frac{1}{2} \iint D_{\chi}\left(\mathbf{r}_{j}(z), \mathbf{r}_{i}\left(z^{\prime}\right)\right) d z d z^{\prime} \\
& -\frac{1}{2} \iint D_{\chi}\left(\mathbf{r}_{j}(z), \mathbf{r}_{j}\left(z^{\prime}\right)\right) d z d z^{\prime} .
\end{aligned}
$$

In order to perform the integrations $C_{n}$ as function of height must be known. Since this is normally not the case we approximate $C_{n}$ to be constant up to an effective tropospheric height $h$ and zero above. The values of $C_{n}$ and $h$ will then be the values for which this approximations agree best with real data; see [14]. Using this approximation, Emardson and Jarlemark [9] made a series expansion of the terms in (5) in the sum of the mapping functions, the product of the mapping functions and the angle between the directions. The result can be written as

$$
\left\langle\left(l_{i}-l_{j}\right)^{2}\right\rangle=\sum_{\nu=1}^{12} a(\nu) b_{\nu}
$$

where $a(\nu)$ are constants and $b_{\nu}$ combinations of the mapping functions and the angle angle between the two directions. The definition of those parameters is given in [9]. The values of $C_{n}$ and $h$ used to obtain $a(\nu)$ are given in [14] as $C_{n}=C_{n 0}=$ $2.4 \cdot 10^{-7} \mathrm{~m}^{-1 / 3}$ and $h=h_{0}=1 \mathrm{~km}$. These were determined from radiometer and radiosonde measurements from California, Australia, and Spain. However, these values might not be unique since other values have also been reported [15]. Furthermore, $C_{n}$ and $h$ are site dependent and have seasonal variations (since the parameters depend on the amount of water vapor in the atmosphere). For these reasons we consider both $C_{n}$ and $h$ as unknowns in our analysis. If these parameters are different from $C_{n 0}$ and $h_{0}$, it is easily shown that

$$
\left\langle\left(l_{i}-l_{j}\right)^{2}\right\rangle=\left.\frac{C_{n}^{2}}{C_{n 0}^{2}} \frac{h^{8 / 3}}{h_{0}^{8 / 3}}\left\langle\left(l_{i}-l_{j}\right)^{2}\right\rangle\right|_{0}=\left.k^{2} \cdot\left\langle\left(l_{i}-l_{j}\right)^{2}\right\rangle\right|_{0}
$$

where $\left.\left\langle\left(l_{i}-l_{j}\right)^{2}\right\rangle\right|_{0}$ denotes the value that $\left\langle\left(l_{i}-l_{j}\right)^{2}\right\rangle$ would have had if $C_{n}$ and $h$ were equal to those used in [14].

When measuring the wet delay with a radiometer the effect of the instrument noise must be considered. Let $A$ denote the radiometer noise expressed in delay. Then the (zenith mapped) wet delay measured by the radiometer, $\hat{l}_{i}$, in the direction $i$ (i.e., at elevation angle $\epsilon_{i}$ and azimuth angle $\phi_{i}$ ) is

$$
\hat{l}_{i}=l_{i}+A \text {. }
$$

It is reasonable to assume that the instrumental noise is independent of $l_{i}-l_{j}$, so $\left\langle\left(l_{i}-l_{j}\right) \cdot \Delta A\right\rangle=\left\langle l_{i}-l_{j}\right\rangle\langle\Delta A\rangle$. In [16], it was reported that there in general are no large long-lived gradients in the wet delay (they were in general less than $1 \mathrm{~mm}$ ), and when large gradient occurred it disappeared rather quickly; hence there is no reason why on average the (zenith mapped) wet delay in one direction should be higher than that in another, i.e., $\left\langle l_{i}-l_{j}\right\rangle=0$. This gives

$$
\left\langle\left(\hat{l}_{i}-\hat{l}_{j}\right)^{2}\right\rangle=\left\langle\left(l_{i}-l_{j}\right)^{2}\right\rangle+\left\langle(\Delta A)^{2}\right\rangle .
$$

The radiometer noise originate from two different sources with different time scales. Over long time scales, hours to days, systematic errors are caused by nonperfect tip-curve calibration [17], [18]. This component has an elevation dependence since it varies with the difference between the observed sky brightness temperature and the reference loads in the radiometer. Over time scales from seconds to a few minutes, relevant in this study, we assume that this component does not contribute to the instrument variance. The other component is the white noise described by [19]

$$
\Delta T=\kappa \frac{T_{\text {sys }}}{\sqrt{\tau \cdot \beta}}
$$

where $\kappa$ is a constant dependent on the type of radiometer; $T_{\text {sys }}$ is the system temperature; $\tau$ is the integration time; and $\beta$ is the bandwidth of the radiometer. The system temperature is in our case dominated by the receiver noise temperatures $(\approx 500-600 \mathrm{~K})$. The variability in the external contribution to $T_{\text {sys }}$ is typically $<100 \mathrm{~K}$ in our climate, when elevation angles above $20^{\circ}$ are used, and observations during rain and high liquid water conditions (high atmospheric opacities) are ignored. The WVR data used in this study are acquired when the zenith liquid water content is less than $0.3 \mathrm{~mm}$. In addition to the above an effect to be considered is appearing when expressing radiometer noise in equivalent path length units. This is due to the retrieval algorithm used to calculate the path length from the observed 
sky brightness temperatures. We use the method of linearized sky brightness temperatures [17] and over time scales of a few minutes and low opacity conditions the variability of this term can be ignored.

In the following we assume that the radiometer noise is the same in every direction, although we note that for future improvements of the model this assumption may turn out to be a limiting factor, depending on the site, season, and the range of elevation angles used. A constant radiometer noise, independent on the direction of the observation, means that we will have an elevation dependence in the noise according to $A(\epsilon)=$ $B / m(\epsilon)$, if the measured equivalent zenith wet delays are used. Inserting this relation into (9) we obtain

$$
\left\langle\left(\hat{l}_{i}-\hat{l}_{j}\right)^{2}\right\rangle=\left.k^{2} \cdot\left\langle\left(l_{i}-l_{j}\right)^{2}\right\rangle\right|_{0}+\left(m_{i}^{-2}+m_{j}^{-2}\right) \cdot \operatorname{Var}[B]
$$

\section{Simulations}

We first assess the model in (11) through simulations. There are two unknowns in the model, $k^{2}$ and $\operatorname{Var}[B]$ which we need to solve for in the analysis. Since the model deals with expectation values we need a certain amount of data in order to have sufficient statistics to retrieve the two unknowns. After having solved for the unknowns we want to test the validity of the model by checking if the residuals are small, which of course also requires a certain amount of data. Therefore, the purpose of the simulations is to investigate how much data we need to use in order to be able to test the validity of the model.

First we simulated observations of $\hat{l}_{i}-\hat{l}_{j}$ for known values of $k^{2}$ and $\operatorname{Var}[B]$. Due to fact that $\left\langle l_{i}-l_{j}\right\rangle=0$, the variance of $l_{i}-l_{j}$ is $\operatorname{Var}\left[l_{i}-l_{j}\right]=\left\langle\left(l_{i}-l_{j}\right)^{2}\right\rangle$, which can be calculated using the model (given a value of $k$ ). If we assume that $l_{i}-l_{j}$ has a Gaussian distribution (which probably is a good approximation), we can get a simulated value of $l_{i}-l_{j}$ by just generating a Gaussian random number having this variance. The radiometer noise was simulated by generating white Gaussian noise with variance $\left(m_{i}^{-2}+m_{j}^{-2}\right) \operatorname{Var}[B]$. Although we express the radiometer noise in units of path length it is worth noting that an equivalent zenith $\mathrm{rms}$ instrument noise of $1 \mathrm{~K}$ in both channels correspond to an rms of $0.76 \mathrm{~cm}$ in path length, or $0.58 \mathrm{~cm}^{2}$ in $\operatorname{Var}[B]$, assuming that the noises in the two channels are independent. Our simulations are in the range $0.04-1.0 \mathrm{~cm}^{2}$ for $\operatorname{Var}[B]$ corresponding to an equivalent zenith rms noise in antenna temperatures of $0.2-1.3 \mathrm{~K}$. We simulated observations for a large number of pairs of directions. The pairs of directions used were the same as for a typical day of real data (i.e., all pairs possible where the WVR observations in each pair are separated by less than $300 \mathrm{~s}$ in time). When the simulated values of $\left(\hat{l}_{i}-\hat{l}_{j}\right)^{2}$ had been obtained a least square fit was made to these values in order to obtain $k^{2}$ and $\operatorname{Var}[B]$ in (11). We then compare the estimated with the initially assumed values.

In Fig. 2 the results of the simulations are displayed. Plotted are the simulated values of $\left\langle\left(\hat{l}_{i}-\hat{l}_{j}\right)^{2}\right\rangle$ including atmospheric variation and instrumental noise together with the model prediction of the atmosphere only and the resulting residual after solving for $k^{2}$ and $\operatorname{Var}[B]$ as function of the difference in angle. Plotted are the data where one of the directions is in the zenith
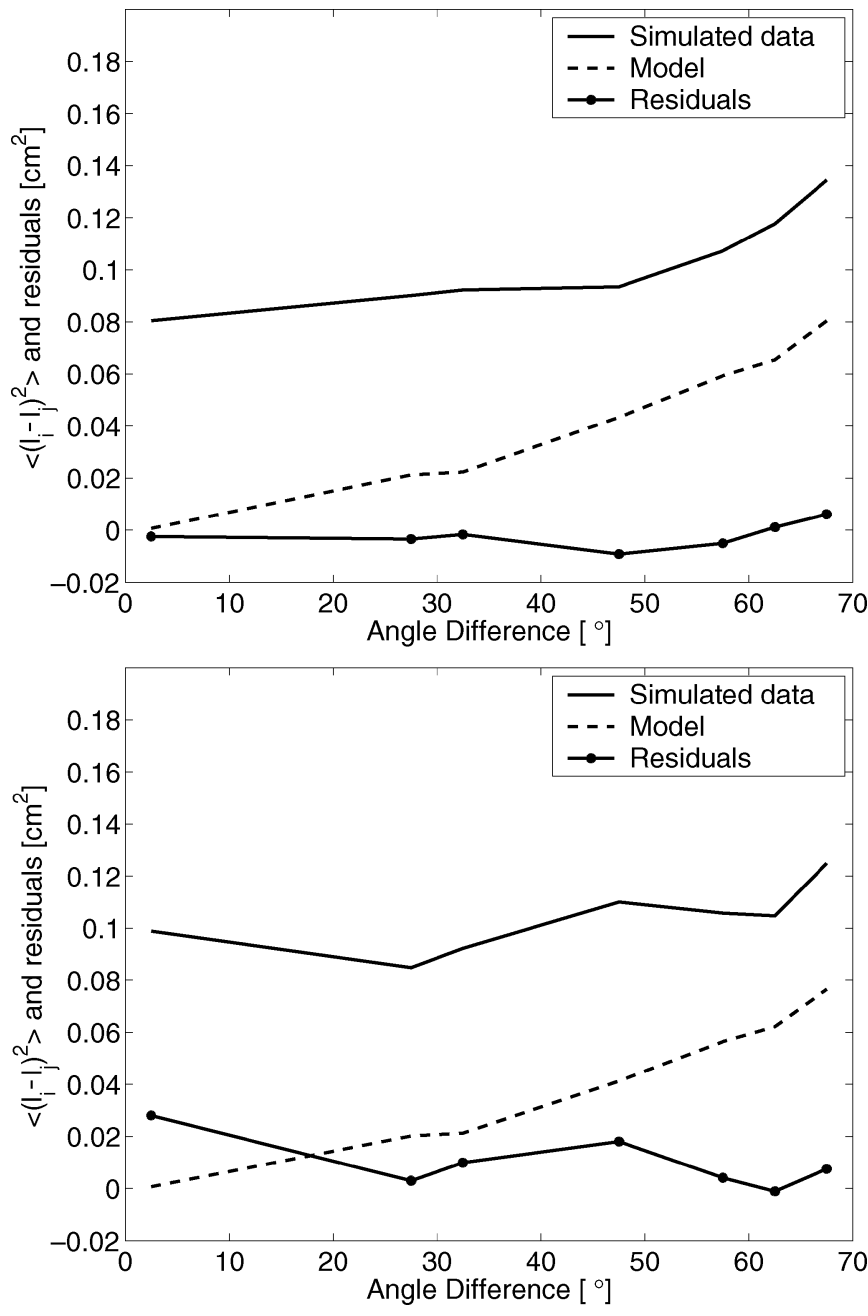

Fig. 2. Result from two simulations. Displayed are the simulated $\left\langle\left(l_{i}-l_{j}\right)^{2}\right\rangle$ (solid line), the model prediction $k^{2} .\left.\left\langle\left(l_{i}-l_{j}\right)^{2}\right\rangle\right|_{0}$ (dotted line) and the residual $\left\langle\left(l_{i}-l_{j}\right)^{2}\right\rangle-\left.k^{2} \cdot\left\langle\left(l_{i}-l_{j}\right)^{2}\right\rangle\right|_{0}-\left(m_{i}^{-2}+m_{j}^{-2}\right) \cdot \operatorname{Var}[B]$ (solid line with dots) as a function of the angle difference. The variance of the noise $B$ is $0.04 \mathrm{~cm}^{2}$ and $k^{2}=3$ for both plots. The simulation periods are one day long for the upper and $6 \mathrm{~h}$ long for the lower plot, respectively. For simplicity, only data including one observation in the zenith direction are shown, but all data are used in the least square fit.

direction, meaning that the angle difference for the data shown is the difference in elevation. A mean is taken over all observations in $5^{\circ}$ intervals to obtain the expectation values.

The top plot in Fig. 2 is for a simulation where the same number of observations (of differences in equivalent zenith wet delay) as from a real on day of observations (containing about 110000 observations). As we can see, the residuals are close to zero. However, when studying real data from one full day the results will probably not be that good. This is because in the simulations we have assumed that all observations $\left(\hat{l}_{i}-\hat{l}_{j}\right)$ are independent, something which i reality might not hold. Observations of $\hat{l}_{i}-\hat{l}_{j}$ at two different epochs (using the same two directions $i$ and $j$ at both epochs) will not be independent if the time between the epochs is small. This will decrease the information we get from the data, i.e., the same effect as if we would decrease the number of observations. The bottom plot in Fig. 2 is using the same number of observations as we get from $6 \mathrm{~h}$ of real data (about 27500 observations, here also $k^{2}=3$ and 

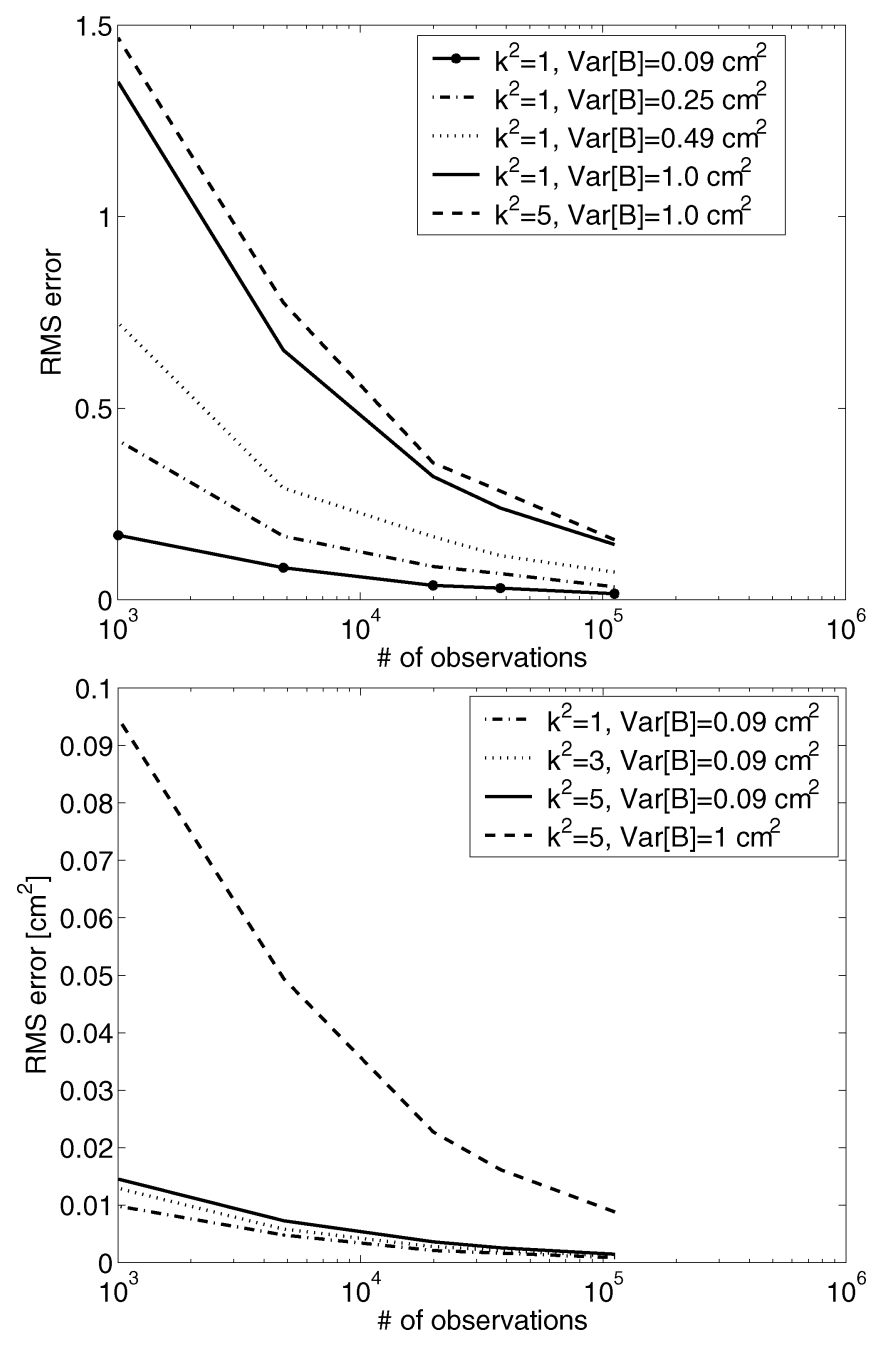

Fig. 3. RMS error of the retrieved values of (top) $k^{2}$ and (bottom) $\operatorname{Var}[B]$ as a function of the number of simulated observations for different values of $k^{2}$ and $\operatorname{Var}[B]$.

$\left.\operatorname{Var}[B]=0.04 \mathrm{~cm}^{2}\right)$. As we see the residuals have increased slightly. On average the residuals (using all simulated data) increased from about $0.014-0.027 \mathrm{~cm}^{2}$ when using $24-$ and $6-\mathrm{h}$ datasets, respectively.

The simulations show that the values of $k^{2}$ and $\operatorname{Var}[B]$ can be obtained with a rather good accuracy, the error of the values of $k^{2}$ obtained in the simulations was about $5 \%$ to $10 \%$ for realistic noise levels (standard deviation of a few millimeters) and the noise level was obtained with even better accuracy (the error was less than 5\%). However, when the noise was in the order of a few centimeters the estimations became worse. Also when reducing the number of observations the error in the estimations increased. In Fig. 3 the RMS errors of the estimation of $k^{2}$ and $\operatorname{Var}[B]$ are plotted as a function of the number of observations used and for a few different values of $k^{2}$ and $\operatorname{Var}[B]$. As seen the RMS errors are larger for larger values of $k^{2}$ and $\operatorname{Var}[B]$. For each simulated combination of $k^{2}$ and $\operatorname{Var}[B], 200$ simulated realizations were used. For each setup the RMS errors were then calculated from the retrieved values of $k^{2}$ and $\operatorname{Var}[B]$.

In the simulations as well as when investigating the real data we assumed that $k^{2}$ and $\operatorname{Var}[B]$ are constant. This might not be true when using real data. To investigate the impact a time
TABLE I

Estimated VALUeS of $k^{2}$ FOR DifFerENT MAXIMUM Times Between THE OBSERVATIONS AND DIFFERENT DAYS

\begin{tabular}{l||c|c|c|c|c|c|c}
\hline & $60 \mathrm{~s}$ & $100 \mathrm{~s}$ & $150 \mathrm{~s}$ & $200 \mathrm{~s}$ & $300 \mathrm{~s}$ & $500 \mathrm{~s}$ & $700 \mathrm{~s}$ \\
\hline \hline 030502 & 1.10 & 1.36 & 1.25 & 1.19 & 1.29 & 1.25 & 1.33 \\
030511 & 1.60 & 1.72 & 1.80 & 1.74 & 1.79 & 1.78 & 1.79 \\
030520 & 3.11 & 3.36 & 3.38 & 3.44 & 3.45 & 3.52 & 3.70 \\
030526 & 1.43 & 1.47 & 1.60 & 1.72 & 1.62 & 1.68 & 1.73 \\
030601 & 1.69 & 1.66 & 1.70 & 1.80 & 1.71 & 1.75 & 1.77 \\
030610 & 2.92 & 3.06 & 3.20 & 3.40 & 3.31 & 3.34 & 3.48 \\
030619 & 3.72 & 3.62 & 3.61 & 3.67 & 3.58 & 3.58 & 3.97 \\
\hline
\end{tabular}

TABLE II

ESTIMATED VARIANCE OF THE NOISE FOR DIFFERENT MAXIMUM Times BETWEEN OBSERVATIONS AND DIFFERENT DAYS. THE UNIT IS SQUARE CENTIMETERS

\begin{tabular}{c||c|c|c|c|c|c|c}
\hline & $60 \mathrm{~s}$ & $100 \mathrm{~s}$ & $150 \mathrm{~s}$ & $200 \mathrm{~s}$ & $300 \mathrm{~s}$ & $500 \mathrm{~s}$ & $700 \mathrm{~s}$ \\
\hline \hline 030502 & 0.007 & 0.002 & 0.005 & 0.007 & 0.009 & 0.016 & 0.022 \\
030511 & 0.013 & 0.013 & 0.009 & 0.012 & 0.016 & 0.024 & 0.030 \\
030520 & 0.028 & 0.027 & 0.024 & 0.028 & 0.031 & 0.045 & 0.053 \\
030526 & 0.014 & 0.015 & 0.016 & 0.015 & 0.017 & 0.022 & 0.025 \\
030601 & 0.002 & 0.002 & 0.004 & 0.003 & 0.005 & 0.008 & 0.010 \\
030610 & 0.020 & 0.019 & 0.020 & 0.018 & 0.023 & 0.035 & 0.044 \\
030619 & 0.019 & 0.022 & 0.025 & 0.026 & 0.032 & 0.046 & 0.056 \\
\hline
\end{tabular}

varying $k^{2}$ or $\operatorname{Var}[B]$ would have on our result we made simulations when $k^{2}$ and $\operatorname{Var}[B]$ varied in time (the types of variations investigated were linear, sinusoidal and discrete). The retrieved values of $k^{2}$ and $\operatorname{Var}[B]$ (which were estimated as constant) turned out to be equal to their average values, as could be expected. The accuracy was about the same as when using constant values for $k^{2}$ and $\operatorname{Var}[B]$.

\section{EXPERIMENTAL RESULTS}

\section{A. Maximum Time Between Observations}

The model (11) gives the correlation between the wet delays in two different directions at the same time epoch. Since the WVR can only measure in one direction at a time we cannot compare the wet delay in two different directions at the same time. Therefore, when using WVR data to test the model, the time between the two observations being compared must be short enough for the temporal variability in the wet delays to be negligible.

To assess the impact of the temporal differences we used WVR data from a whole day and calculated all possible squared differences of (zenith mapped) wet delay for observations being less than $\Delta t_{\max }$ in time from each other. Then a least square fit was made to obtain the values of $k^{2}$ and $\operatorname{Var}[B]$ in (11) for different values of $\Delta t_{\max }$ and using data from different days in May and June of 2003. Days when more than $25 \%$ of the measurements had been discarded in the WVR processing (because of liquid water content larger than $0.3 \mathrm{~mm}$ ) were not considered. The results from some of the investigated days can be seen in Tables I and II. If the change in the wet delay can be neglected the obtained values of $k^{2}$ and $\operatorname{Var}[B]$ will be independent of the choice of $\Delta t_{\max }$. As seen in Tables I and II there are no dramatic differences between the obtained values using the different maximum time differences, only a slight increase especially in the noise estimate can be observed for $\Delta t_{\max }>300 \mathrm{~s}$. Therefore we choose in the following investigations to let $\Delta t_{\max }$ be $300 \mathrm{~s}$. A higher value could have probably been chosen for $\Delta t_{\max }$ for some days (days 

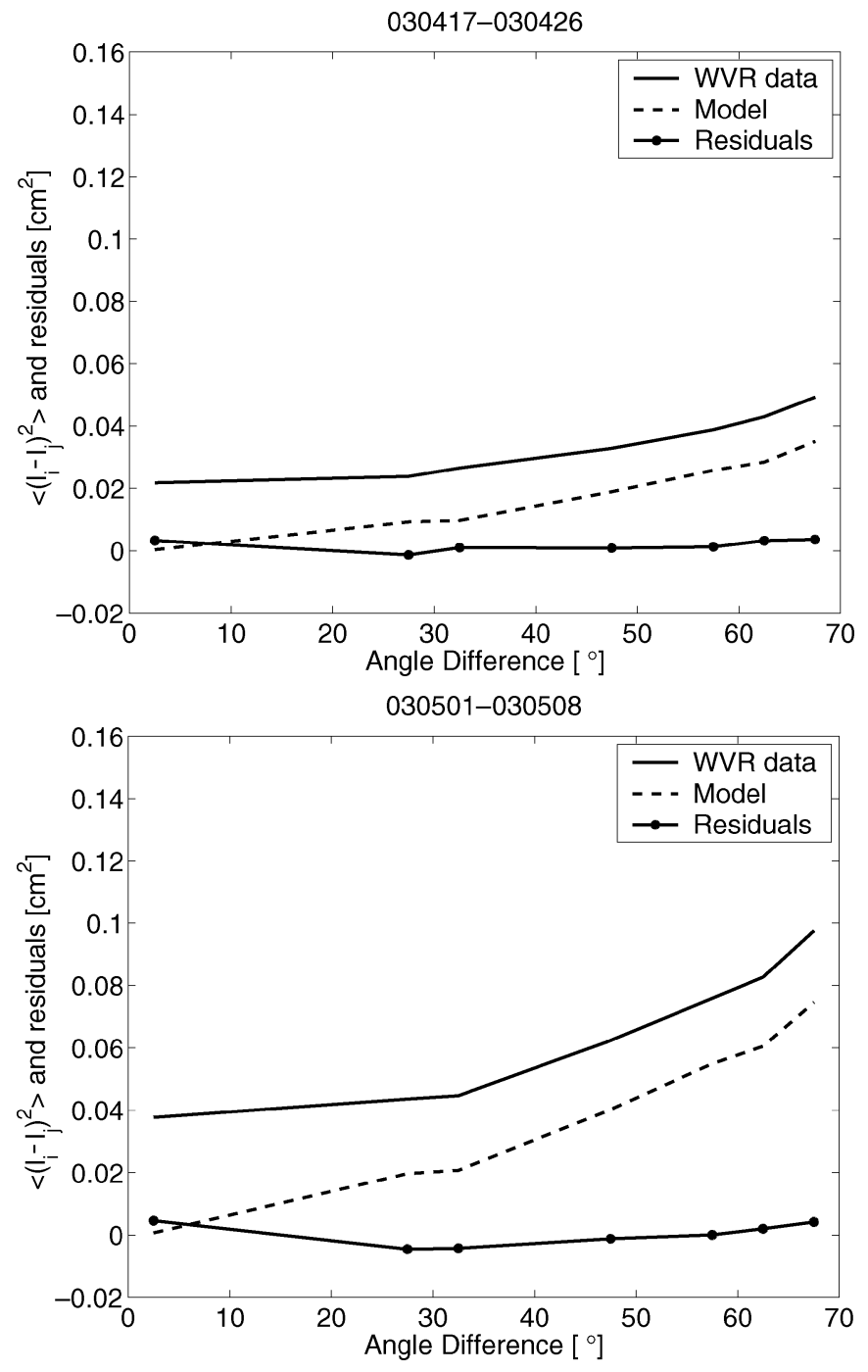

Fig. 4. Result from two periods in 2003. Displayed are the same quantities as described in the caption of Fig. 2 (except that real data are used instead of simulated data).

with a low temporal variability) but this was not done in order to have the datasets containing approximately the same number of observations and having the same set of directions.

From Tables I and II, we note that there is a correlation between the estimated values of $k^{2}$ and $\operatorname{Var}[B]$. Of course, such correlations should not be present. This may be due to residual effects of liquid water in the atmosphere and/or other nonwhite processes in the instrumental noise. The results in Tables I and II are obtained using data where the equivalent zenith liquid water content inferred from the observations is low, less than $0.3 \mathrm{~mm}$. If we allow for a higher liquid water content in the observations we see that the correlation become stronger. The reasons for this effect is that the retrieval algorithm for the wet delay using radiometer data is less accurate when the liquid water content is high and that the model does not describe the atmosphere as well when there are dense liquid water clouds present.

\section{B. Comparison Between Model and Real Data}

In Fig. 4 the results from two periods in 2003 are displayed. The chosen data had no liquid water content larger than $0.3 \mathrm{~mm}$, an assumption used by the WVR data retrieval algorithm (for
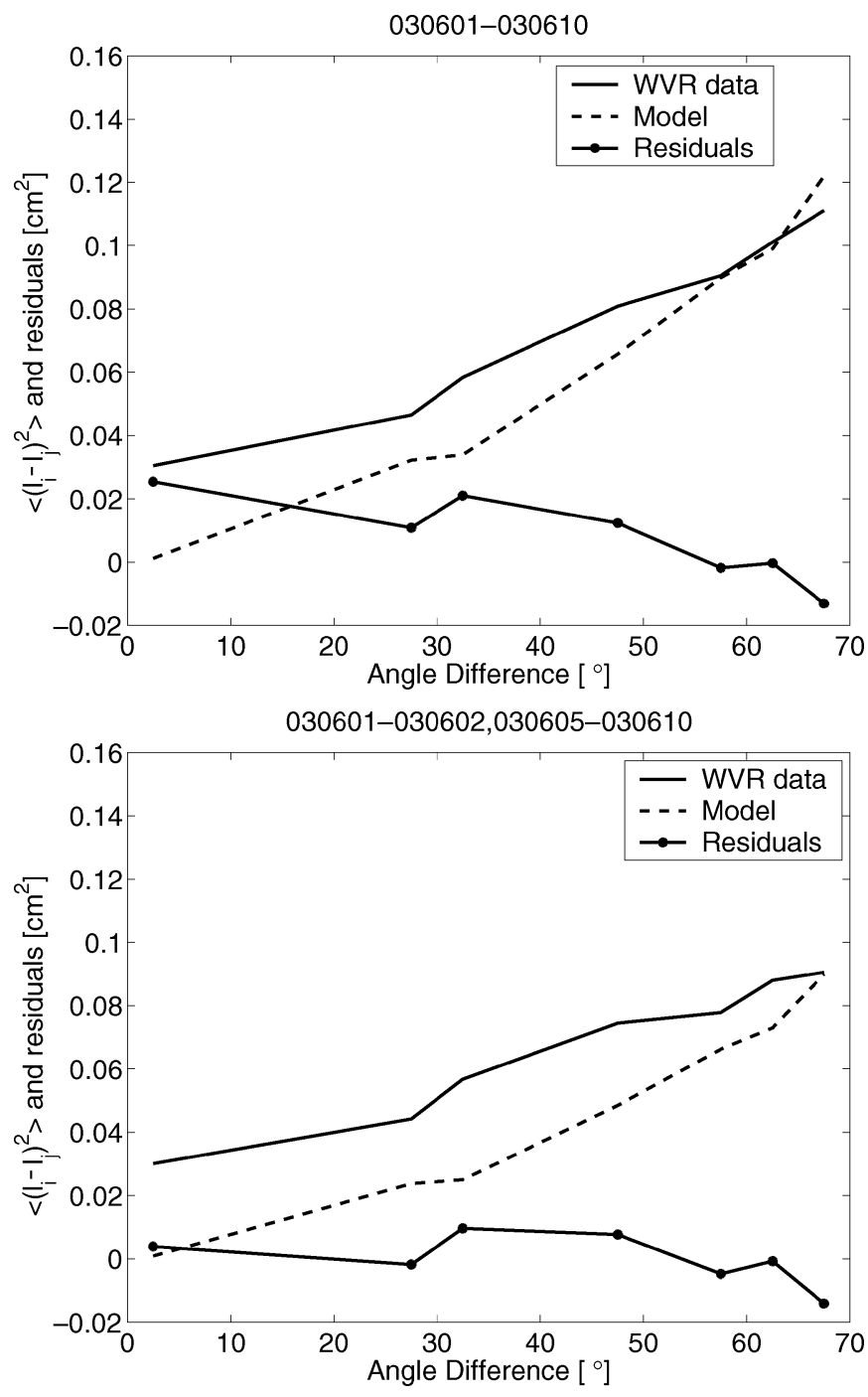

Fig. 5. Result from one period in 2003. The bottom plot is with the data from two of the days removed (see text). Displayed are the same quantities as described in the caption of Fig. 2 (except that real data are used instead of simulated data).

further discussion on the subject, see [11] and [20]). Since the WVR during this time was only making very few measurements in the zenith direction, the data points plotted in Fig. 4 are only a small part of the total amount of data, hence the statistics may not be sufficient to draw any conclusions only from the plot.

The results in Fig. 4 show small residuals. Calculating the mean of all residuals based on sufficient statistics (more than 500 measurements included in each estimation step) gave values of $0.012 \mathrm{~cm}^{2}$ for the April period and $0.023 \mathrm{~cm}^{2}$ for the May period. These were larger for lower elevations, which may be explained by the fact that the beamwidth of the WVR is nonzero and this may cause the effective elevation angle of the beam to be slightly lower at low elevations (since observations at lower elevations have a longer path through the atmosphere). Although in general the agreement between the data and the model was good we had periods with a worse performance present. In Fig. 5 (top), the period June 1-10, 2003 is plotted and as it can be seen the residuals are somewhat larger. Calculating $k^{2}$ and $\operatorname{Var}[B]$ for the individual days in this period revealed two days with a very high $k^{2}$ value. If $k^{2}$ (and hence $C_{n}$ ) is large it means that 
the atmosphere is very variable not only in space but also in time. Hence, the maximum time allowed between observation determined in Section IV-A might be too large for these days. Also, it might be suspected that during these days the atmosphere was not described very well by the model. Removing these two days from the analysis gave a better agreement with the model as seen in the bottom plot in Fig. 5 .

\section{WVR-Related Issues}

In the beginning of 2003 the Onsala WVR was upgraded. In order to detect errors due to instrumental uncertainties, a comparison between the data recorded before and after the upgrade was made. The estimated mean standard deviations of the noise $(\sqrt{\operatorname{Var}[B]})$ was $2.9 \mathrm{~mm}$ for the investigated data from the period 2000-2002 and $1.9 \mathrm{~mm}$ for the 2003 data.

It should, however, be noted that the 2003 data are mainly from the period April to June, and since the atmospheric variability depends on the season this may have influenced the result. However, the 2000-2002 data show that the noise reaches its minimum in February and its maximum in August, and since the 2003 data are from a period in the middle, we do not expect this effect to be dominant.

Before upgrading the WVR, it occasionally produced measurements which were incorrect (they clearly deviated from the rest of the data and were probably caused by a failing analog to digital converter). Such incorrect data can easily be identified and removed if the deviation from the time series of equivalent zenith wet delay is large, but not if the atmosphere itself shows a large variability. To estimate the influence of this effect on our results a very simple model was used. Removing all data having a large deviation from nearby observations, will leave only those incorrect observations which could be approximately described by what would have been measured if the radiometer had worked correctly and the measured wet delays was disturbed by white noise after being mapped to zenith. To account for this, we modify (11) by introducing such a white noise term $W$

$$
\begin{aligned}
\left\langle\left(\hat{l}_{i}-\hat{l}_{j}\right)^{2}\right\rangle= & \left.k^{2} \cdot\left\langle\left(l_{i}-l_{j}\right)^{2}\right\rangle\right|_{0} \\
& +\left(m_{i}^{-2}+m_{j}^{-2}\right) \cdot \operatorname{Var}[B]+\operatorname{Var}[W]
\end{aligned}
$$

After making a least square fit of the data in (12) and obtaining $k^{2}, \operatorname{Var}[B]$ and $\operatorname{Var}[W]$, we observed that most of the data recorded before the upgrade gave a significant positive value of $\operatorname{Var}[W]$. Also the deviation between the model and the real data was clearly smaller with the $W$ term included. The retrieved values for $\operatorname{Var}[B]$ and $k^{2}$ were also somewhat smaller (about $0.03 \mathrm{~cm}^{2}$ and 0.8 , respectively) when including the $W$ term. We also observed that the impact of the incorrect observations tended to be larger in the summer. One reason for this may be that incorrect observations are more easy to identify during the winter since the atmosphere then in general is less variable. It may also be an effect of inaccurate corrections for liquid water in clouds since convective activity is more common in the summer.

If the same analysis is applied on the data recorded after the upgrade the obtained values of $\operatorname{Var}[W]$ are in most cases small and even negative. The inclusion of the $W$ term did not give any significant reduction of the deviation of the data from the model.
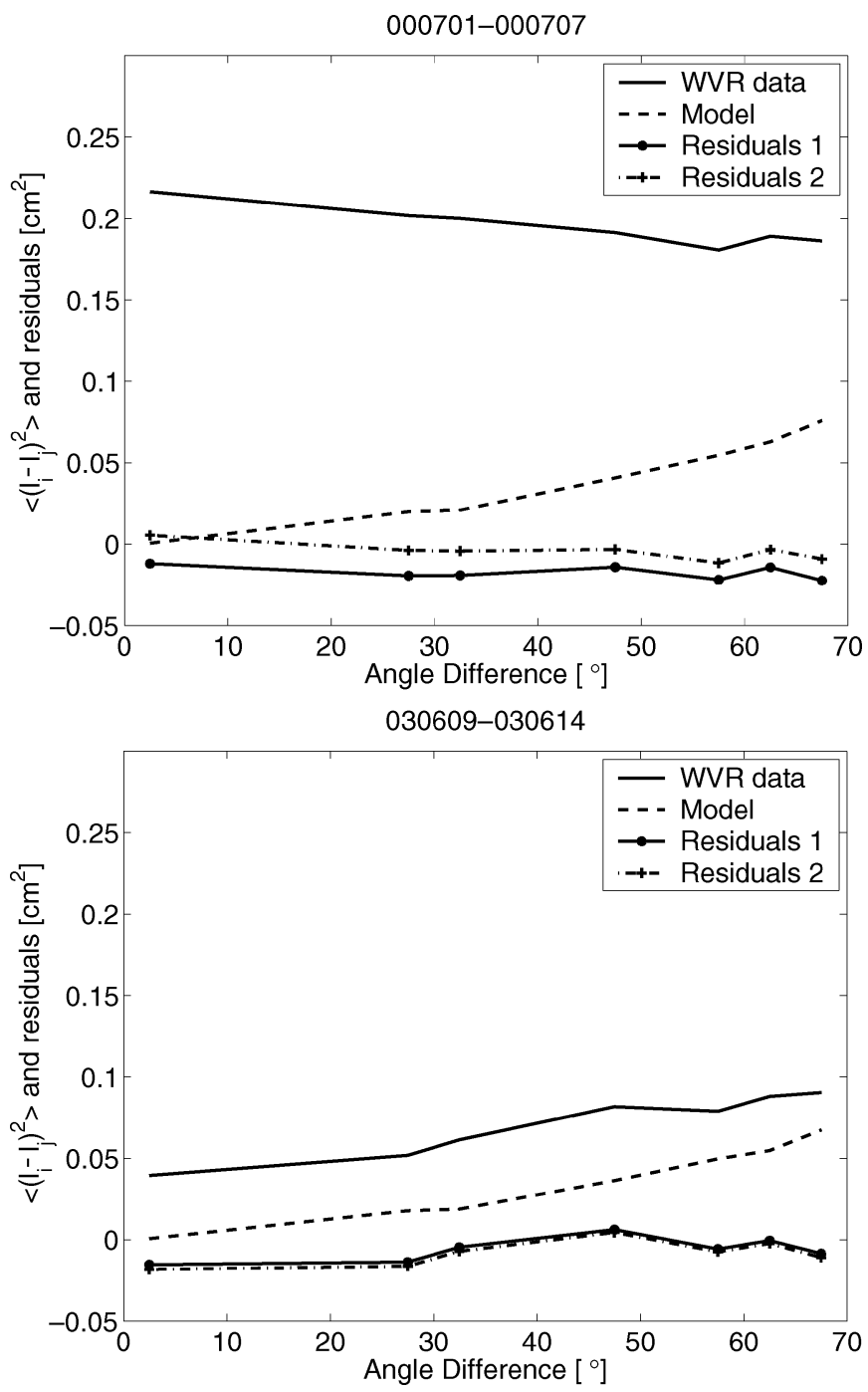

Fig. 6. Comparison of the results using the model two models [(11) and (12)]. Displayed are the same quantities as described in the caption of Fig. 2 (except that real data are used instead of simulated data). The two different residuals are using the model in (11) (residuals 1) and (12) (residuals 2). Note that in the lower plot the two residuals are almost equal.

In Fig. 6 the results from two periods, one before and one after the upgrade, are plotted. Displayed are both the error using the original model (11) and the model with the $W$ term (12). We can see that for the period before the upgrade the model in (12) gives smaller residuals than the model in (11), while there is hardly any difference in the residuals for the period after the upgrade. When looking at all residuals we noted a significant decrease (the mean of the residuals decreased from $0.025 \mathrm{~cm}^{2}$ to $0.022 \mathrm{~cm}^{2}$ ) for the year 2000 period, while there was almost no decrease in the residuals for the year 2003 period (about $0.028 \mathrm{~cm}^{2}$ in both cases). From this we may conclude that the number of incorrect observations after the upgrade has been reduced.

\section{Seasonal Variability of $C_{n}$}

In Fig. 7, the values of $k$ obtained from the WVR data covering the period 1992-2002 are plotted as a function of the season, together with a simple sinusoidal fit made to the data. Each datapoint represents the $k$-value derived from one dataset. Before July 2000 a dataset typically included 2-3 days of data. 


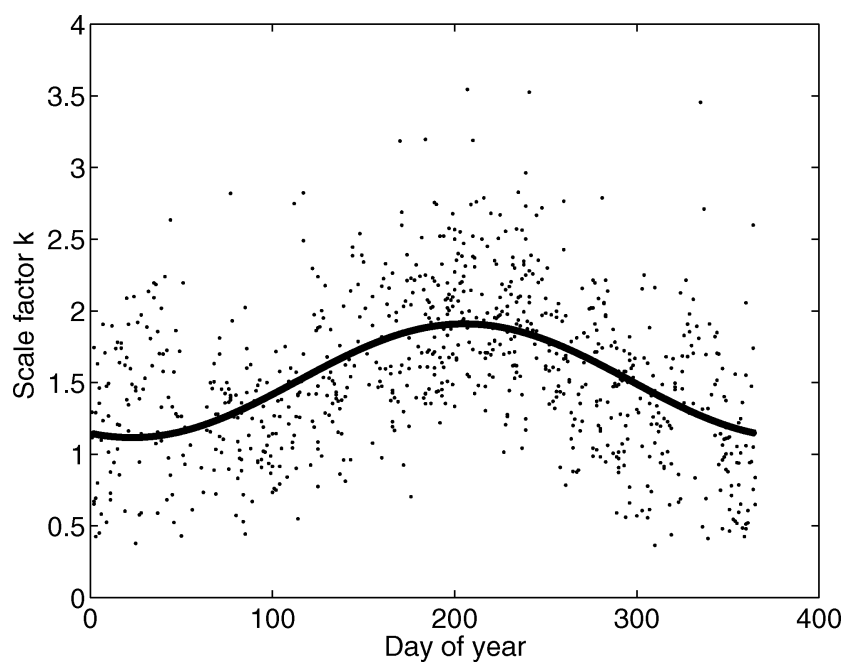

Fig. 7. Seasonal variation of $k$ derived from the radiometer data using the model, together with a sinusiondal fit. The data used are from the period 1992-2002.

After this date each dataset equals one day. In total approximately 1400 days of WVR data are included. In order not to have the data being affected by, for example, diurnal effects we only used data sets not containing any large gaps (caused by data being removed due to high liquid water content) in the data ( $>300 \mathrm{~s}$ ). Also, we could not use data from periods when the WVR only had been measuring in the zenith direction. We see that $k$ reaches its minimum in the end of January and its maximum in the end of July, following well the trend of the total amount of water vapor. The variation is about $\pm 26 \%$ relative to its average value.

From (7) we see that $k$ is proportional to $C_{n} h^{4 / 3}$. Hence, the variation of $k$ can be a consequence of a variation in $C_{n}$ or a variation in $h$. In [14], it was noted that the effective tropospheric height was about half the scale height of the tropospheric water vapor $H_{s c l}$. This scale height is defined by the average profile of the wet refractivity when it is described by

$$
\chi(z)=\chi_{0} \cdot \exp \left(-\frac{z}{H_{s c l}}\right) .
$$

In order to investigate how much the effect a varying $h$ can have on our measurements, we used seven years of data from radiosondes launched at the Gothenburg-Landvetter Airport located $37 \mathrm{~km}$ away from the Onsala Space Observatory. We estimated the scale height $H_{s c l}$ and obtained a mean value of $\sim 2040 \mathrm{~m}$ and a seasonal variation of $\pm 190 \mathrm{~m}$ (with maximum/minimum at the same epoch as $k$ ). Assuming that $h$ is proportional to $H_{s c l}$, this will introduce a seasonal variation in $k$ of about $13 \%$ relative to its mean value. This is significantly lower than the seasonal variation shown in Fig. 7 (which is $\pm 26 \%$ relative to the mean value) indicating that there is still some seasonal variation due to $C_{n}$ (about $\pm 12 \%$ ).

It is possible that the result is affected by the intermittent problems with the analog-to-digital conversion in the WVR before 2003 (see Section IV-C). However, it is likely that this effect will mainly cause the average value of $k$ to be lower, but the relative seasonal variation will not be affected much. If the modified model (12) is used to retrieve $k$ instead, the seasonal variation is actually larger than what was obtained when $k$ was retrieved from (11), about $\pm 28 \%$ instead of about $\pm 26 \%$. The average value of $k$ also decreased somewhat, from about 1.51 to about 1.34 .

Our results on the variation agree with the results presented in [21] and [22], where a $20 \%$ to $50 \%$ seasonal variation of $C_{n}$ was reported. These estimates were obtained from investigations of the temporal variations of the zenith wet delay measured by WVR and GPS, and assuming that no variations in $h$ occurred.

\section{CONCLUSION}

We demonstrated that the agreement between the presented slant delay correlation model and the WVR data is rather good, the residuals being $0.01-0.04 \mathrm{~cm}^{2}$ (larger residuals for larger $k$, as could be expected). It should be emphasized that (6), which was used to calculate the model prediction, is only an approximation which may deviate somewhat from what would be predicted by (5).

In this work we have only used days where the liquid water content in the atmosphere is low since the WVR retrieval algorithm introduce an extra noise when the liquid water content is high. Hence, we have not validated the model for conditions with high amount of liquid water in the atmosphere or in rain.

Since the model (6) seems to describe the WVR data well, we can conclude that the model for the structure function in (3) can also be useful. One possible application of the model (6) would be to use it as a constraint when estimating slant wet delays directly from GPS data (note that a different model for the instrumental noise should be used in this case). Since the equation gives the correlation between the refractivity at two points in space it can also be used to provide constraints to the GPS tropospheric tomography [5] (this of course requires $C_{n}$ to be known with some accuracy).

Another possible application of this work is to use the model in (12) to obtain information on the WVR stability in terms of instrumental noise. We obtained a clear indication of an improvement of the radiometer measurement uncertainty from an upgrade of the data acquisition unit using a simple model for the instrument noise. Although this was a useful verification of an expected result, further work may consider to investigate slightly more advanced models. For example, the effect of an elevation dependence in the radiometer noise can be included in the model. We foresee that such models can be used operationally in order to monitor the instrumental performance over long time periods.

\section{ACKNOWLEDGMENT}

The authors are grateful to P. Jarlemark, K.- $\mathrm{A}$. Johansson, and B. Stoew for their dedicated work to keep the microwave radiometer operating for most of the time during the last decade. The authors also thank the four anonymous reviewers for pointing out many issues that needed clarifications.

\section{REFERENCES}

[1] V. I. Tatarskii, The Effects of the Turbulent Atmosphere on Wave Propagation. Jerusalem, Israel: Israel Program for Scientific Translations, 1971. 
[2] H. Vasseur, "Prediction of tropospheric scintillation on satellite links from radiosonde data," IEEE Trans. Antennas Propagat., vol. 47, no. 2, pp. 293-301, Feb. 1999.

[3] P. Fang, M. Bevis, Y. Bock, S. Gutman, and D. Wolfe, "GPS meteorology: Reducing systematic errors in geodetic estimates for zenith delay," Geophys. Res. Lett., vol. 25, pp. 3583-3586, 1998.

[4] A. Flores, J. V.-G. de Arellano, L. P. Gradinarsky, and A. Ruis, "Tomography of the lower troposphere using a small dense network of GPS receivers," IEEE Trans. Geosci. Remote Sens., vol. 39, no. 2, pp. 439-447, Feb. 2001.

[5] L. Gradinarsky and P. Jarlemark, "Ground-based GPS tomography of water vapor: Analysis of simulated and real data," J. Meteorol. Soc. Japan, vol. 82, pp. 551-560, 2004.

[6] C. Rocken, T. V. Hove, J. Johnson, F. Solheim, and R. Ware, "GPS/STORM-GPS sensing of atmospheric water vapor for meteorology," J. Atmos. Oceanic Technol., vol. 12, pp. 468-478, 1995.

[7] P. Tregoning, R. Boers, D. O’Brien, and M. Hendy, "Accuracy of absolute precipitable water vapor estimates from GPS observations," J. Geophys. Res., vol. 103, pp. 28 701-28 710, 1998.

[8] T. R. Emardson, G. Elgered, and J. M. Johansson, "Three months of continuous monitoring of atmospheric water vapor with a network of global positioning system receivers," J. Geophys. Res., vol. 103, no. D2, pp. $1807-1820,1998$

[9] T. R. Emardson and P. O. J. Jarlemark, "Atmospheric modeling in GPS analysis and its effect on the estimated geodetic parameters," J. Geodesy, vol. 73, p. 322, 1999.

[10] J. Braun, C. Rocken, and J. Liljegren, "Comparisons of line-of-sight water vapor observations using the global positioning system and a pointing microwave radiometer," J. Atmos. Oceanic Technol., vol. 20, pp. 606-612, 2003

[11] G. Elgered and P. O. J. Jarlemark, "Ground-based microwave radiometry and long-term observations of atmospheric water vapor," Radio Sci., vol. 33, pp. 707-717, May 1998.

[12] G. Elgered, "Tropospheric radio-path delay from ground-based microwave radiometry," in Atmospheric Remote Sensing by Microwave Radiometry, M. Janssen, Ed. New York: Wiley, 1993, pp. 215-258.

[13] J. L. Davis, T. A. Herring, I. I. Shapiro, A. E. E. Rogers, and G. Elgered, "Geodesy by radio interferometry: Effects of atmospheric modeling errors of baseline length," Radio Sci., vol. 20, pp. 1593-1607, 1985.

[14] R. Treuhaft and G. Lanyi, "The effect of the dynamic wet troposphere on radio interferometric measurements," Radio Sci., vol. 22, pp. 251-265, 1987.

[15] R. Linfield, "The effect of aperture averaging upon tropospheric delay fluctuations seen with a DSN antenna," NASA, Greenbelt, MD, In Telecommunications and Data Acquisition Progress Report, pp. 1-7, [Online]. Available: http://tmo.jpl.nasa.gov/tmo/progress_report/ 42-124/124A.pdf, vol. 42-124A, 1996.

[16] L. Gradinarsky and G. Elgered, "Horizontal gradients in the wet path delay derived from four years of microwave radiometer data," Geophys. Res. Lett., vol. 27, pp. 2521-2524, 2000.

[17] S.-C. Wu, "Optimum frequencies of a passive microwave radiometer for tropospheric path-length correction," IEEE Trans. Antennas Propagat., vol. AP-27, pp. 233-239, 1979

[18] Y. Han and E. Westwater, "Analysis and improvement of tipping calibration for ground-based microwave radiometers," IEEE Trans. Geosci. Remote Sens., no. 3, pp. 1260-1276, May 2000.

[19] M. Janssen, Atmospheric Remote Sensing by Microwave Radiometry. New York: Wiley, 1993.
[20] L. Gradinarsky, G. Elgered, and Y. Xue, "Using a micro-rain radar to assess the editing of ground-based microwave radiometer data," in $\mathrm{Mi}$ crowave Radiometry and Remote Sensing of the Earth's Surface and Atmosphere, P. Pampaloni and S. Paloscia, Eds: VSP, 2000, pp. 183-191.

[21] S. J. Keihm, "Water vapor radiometer measurements of the tropospheric delay fluctuations at Goldstone over a full year," NASA, Greenbelt, MD, In Telecommunications and Data Acquisition Progress Report, pp. 1-11, [Online]. Available: http://tmo.jpl.nasa.gov/tmo/progress_report/42-122/122J.pdf, vol. 42-122J, 1995.

[22] C. J. Naudet, "Estimation of tropospheric fluctuations using GPS data," NASA, Greenbelt, MD, In Telecommunications and Data Acquisition Progress Report, pp. 1-19, [Online]. Available: http://tmo.jpl.nasa.gov/ tmo/progress_report/42-126/126A.pdf, vol. 42-126A, 1996.

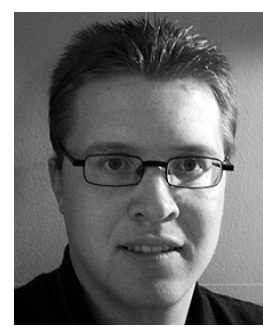

Tobias Nilsson was born on October 7, 1978, in Svenljunga, Sweden. He received the M.Sc. degree in engineering physics from Uppsala University, Uppsala, Sweden, in 2003, and is currently pursuing the $\mathrm{Ph} . \mathrm{D}$. degree at the Onsala Space Observatory, Chalmers University of Technology, Göteborg, Sweden.

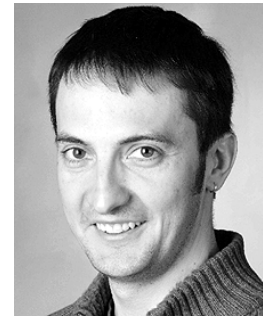

Lubomir Gradinarsky (M'02) was born in Sofia, Bulgaria, in 1970. He received the M.S.E.E and the Ph.D degrees from Chalmers University of Technology, Göteborg, Sweden, in 1996 and 2002, respectively.

$\mathrm{He}$ is presently a Senior Scientist in Sensor Technology at AstraZeneca R\&D Mölndal, Sweden. His research has been focused on remote sensing of the atmosphere using space geodetic techniques and microwave radiometry. Currently his work also includes development of remote sensing techniques for industrial applications.

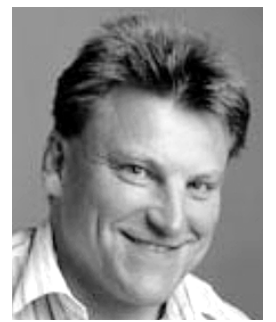

Gunnar Elgered was born in Götene, Sweden, in 1955. He received the M.S.E.E and Ph.D. degrees from Chalmers University of Technology, Göteborg, Sweden, in 1977 and 1983, respectively.

$\mathrm{He}$ is presently a Professor in electrical measurements and chairs the Department of Radio and Space Science at Chalmers University of Technology. His research has been focused on remote sensing of the atmosphere using space geodetic techniques and $\mathrm{mi}$ crowave radiometry. 